\title{
Screen Printed PZT Composite Thick Films
}

\author{
R. A. DOREY, ${ }^{1}$ R. W. WHATMORE, ${ }^{1}$ S. P. BEEBY, ${ }^{2}$ \\ R. N. TORAH, ${ }^{2}$ and N. M. WHITE ${ }^{2}$ \\ ${ }^{1}$ Nanotechnology Group, School of Industrial and Manufacturing Science, \\ Cranfield University, Cranfield, MK43 OAL \\ ${ }^{2}$ Department of Electronics and Computer Science, University of Southampton, \\ Southampton, SO17 1BJ
}

(Received August 8, 2003; in final form January 5, 2004)

Composite film (ComFi) technology has been developed as an alternative technique to achieve thick $(5-100 \mu \mathrm{m})$ films at temperatures compatible with silicon processing. The technique entails producing a composite slurry consisting of PZT powder and a PZT producing sol. The slurry is then deposited onto the substrate, dried and fired at $710^{\circ} \mathrm{C}$. Intermediate sol infiltration and pyrolysis can be used to increase the density and improve the functional properties of the films. A slurry suitable for screen printing has been developed and used to print PZT thick films. The dielectric and piezoelectric properties of the screen printed material are presented. Material properties of the screen printed ComFi material were found to be comparable to, or exceeded, those observed in traditional screen printed materials.

Keywords: Screen printing; PZT; thick film

AIP: 77.22.-d; 77.65.-j; 77.84.-s

\section{INTRODUCTION}

Lead zirconate titanate (PZT) ceramics are of great interest for use in micro electromechanical systems (MEMS) due to the high piezoelectric response that they exhibit. To realise Micro-Electro-Mechanical Systems (MEMS) capable of producing large deflections and high actuation forces it is necessary to incorporate thick piezoelectric elements [1] (typically 5-100 $\mu \mathrm{m}$ ).

Low temperature, thin film technologies (e.g. sputtering, sol gel) require excessive deposition times [2] and are limited in the maximum thickness obtainable due to the build-up of internal stresses during deposition which lead to film cracking [3]. Conversely, conventional bulk ceramic processing with subsequent machining and bonding is both time consuming and wasteful of material. Techniques such as screen printing and electrophoresis 
are less wasteful but still require high temperature processing and refractory substrates [4] which are difficult to integrate into MEMS processing routes [5].

Spin coating composite film (ComFi) technology has successfully been employed in the production of high density, PZT films up to $30 \mu \mathrm{m}$ thick and at temperatures as low as $710^{\circ} \mathrm{C}$ [6]. High thickness films are obtained by repeated deposition but there is a drive to increase the deposition rate of these films. To this end the spin coating powder/sol-gel composite slurry has been modified to produce an ink for use with screen printing technology for the rapid deposition of PZT thick films. The dielectric and piezoelectric properties of the resultant films have been measured and compared to those of films produced using a conventional powder/frit/oil screen printing ink.

\section{EXPERIMENTAL PROCEDURE}

The PZT producing sol was synthesised from lead acetate trihydrate, $\mathrm{Zr}$ isopropoxide, Ti propoxide, and 2-Methoxyethanol [7]. The composite slurry was produced by ball milling the sol, PZT powder $(3 \mathrm{~g}$ powder/ml solferroperm PZ26), dispersant (2 wt\% w.r.t. PZT powder-Kenrich KR55) and sintering aid (4.7 wt\% w.r.t. PZT powder $-0.2 \mathrm{CuO}_{2}-0.8 \mathrm{PbO}$ ) under nitrogen for 24 hours.

$10 \mu \mathrm{m}$ PZT films were deposited by screen printing the composite slurry onto a silicon wafer coated with $60 \mathrm{~nm} \mathrm{ZrO}_{2}, 8 \mathrm{~nm}$ Ti and $200 \mathrm{~nm} \mathrm{Pt}$ $\left(\mathrm{Si} / \mathrm{ZrO}_{2} / \mathrm{Ti} / \mathrm{Pt}\right)$. The screen printed films were dried at $200^{\circ} \mathrm{C}$ and pyrolysed at $450^{\circ} \mathrm{C}$ for 1 minute. The porous films were then infiltrated with sol, spun at $2000 \mathrm{rpm}$, dried, and pyrolysed in order to increase the density of the films. The number of sol infiltration/pyrolysis treatments was varied from 0 to 6 . The films were then sintered at $710^{\circ} \mathrm{C}$ for 30 minutes in an air atmosphere to further densify the films and develop the perovskite structure.

For comparison, $10 \mu \mathrm{m}$ thick PZT films were also produced using a conventional screen printing ink consisting of the same PZT powder and $10 \mathrm{wt} \%$ glass frit dispersed in a pine oil carrier medium. These films were fired at $750^{\circ} \mathrm{C}$ and $890^{\circ} \mathrm{C}$.

Circular $\mathrm{Cr} / \mathrm{Au}$ top electrode structures were deposited by evaporation for electrical characterisation. Capacitance and loss measurements were made using a Wayne Kerr 6425 analyser at $1 \mathrm{kHz}$. The thicknesses of the films were determined from optical and scanning electron microscope (SEM) observations and used to calculate the relative permittivity of the thick films. The films were poled at $130^{\circ} \mathrm{C}$ for 5 minutes using a field of $8 \mathrm{~V} / \mu \mathrm{m}$. 
Following poling the piezoelectric coefficients $\mathrm{d}_{33, \mathrm{f}}$ and $\mathrm{e}_{31, \mathrm{f}}$ were measured using a modified piezometer (TakeControl PM25) [7].

\section{RESULTS AND DISCUSSION}

The relative permittivity of the composite screen printed thick films increased approximately linearly from 300 to 600 when increasing from 0 to 6 sol infiltration/pyrolysis treatments. This is a direct consequence of the increase in the density of the films. The dielectric loss of the material was independent of the change in density and has a value of approximately $1.5 \%$. The $\mathrm{d}_{33, \mathrm{f}}$ piezoelectric coefficient of the thick films increased from approximately 30 to $50 \mathrm{pC} / \mathrm{N}$ when 4 (or more) sol infiltration/pyrolysis treatments were conducted. The initial low piezoelectric coefficients have been attributed to alternative deformation modes of the very low density of the films. The $\mathrm{e}_{31, \mathrm{f}}$ piezoelectric coefficient was found to be approximately $-0.3 \mathrm{C} / \mathrm{m}^{2}$ and independent of the level of sol infiltration/pyrolysis treatments. This value is relatively low in comparison to values obtained from spin coated composite thick films $\left(\mathrm{e}_{31, \mathrm{f}}\right.$ up to $\left.-8 \mathrm{C} / \mathrm{m}^{2}\right)$ and was attributed to the presence of cracks in the surface of the film which act as 'spacers' between islands of active material preventing the transferral of strains across the wafer. Current work is examining the cause of these cracks in an attempt to increase the value of $\mathrm{e}_{31, \mathrm{f}}$.

Table I shows a comparison between the electrical properties of the films produced using the composite sol gel route and those produced using a conventional screen printing ink. Conventional inks require considerably higher processing temperatures to attain comparable functional properties. The enhanced properties of the composite sol gel films are due to the presence of the sol gel derived phase which increases the green density of the film and enhances the densification kinetics due to the small grain size. Furthermore,

TABLE I Comparison of electrical properties of films produced using the composite sol gel ink (6 sol infiltrations) and those produced using the conventional screen printing ink

\begin{tabular}{lccc}
\hline & Composite sol gel & \multicolumn{2}{c}{ Conventional ink } \\
\hline Max processing $\mathrm{T}$ & $710^{\circ} \mathrm{C}$ & $750^{\circ} \mathrm{C}$ & $890^{\circ} \mathrm{C}$ \\
Relative permittivity & 600 & 290 & 615 \\
$\mathrm{~d}_{33, \mathrm{f}}$ piezo. coef. & $50 \mathrm{pC} / \mathrm{N}$ & $20 \mathrm{pC} / \mathrm{N}$ & $20 \mathrm{pC} / \mathrm{N}$ \\
e $_{31, \mathrm{f} \text { piezo. coef. }}$ & $-0.3 \mathrm{C} / \mathrm{m}^{2}$ & $-0.2 \mathrm{C} / \mathrm{m}^{2}$ & $-0.7 \mathrm{C} / \mathrm{m}^{2}$ \\
\hline
\end{tabular}


the absence of large quantities of inactive filler also enhances the functional properties.

\section{CONCLUSION}

$10 \mu \mathrm{m}$ thick films have been produced using a composite sol gel screen printing technique. High density films were obtained following repeated sol infiltration and pyrolysis of the porous screen printed films.

Composite films produced at $710^{\circ} \mathrm{C}$ exhibit dielectric and piezoelectric properties that are comparable to, or greater than, those of films produced at $890^{\circ} \mathrm{C}$ using a conventional powder/frit/oil ink.

\section{REFERENCES}

[1] E. S. Thiele, D. Damjanovic, and N. Setter, "Processing and properties of screen-printed lead zirconate titante piezoelectric thick films on electroded silicon," J. Am. Ceram. Soc. 84(12), 2863-2868 (2001).

[2] N. Setter, "Elecroceramics: looking ahead," J. Euro. Ceram. Soc. 21, 1279-1293 (2001).

[3] Q, F. Zhou, H. L. W. Chen, and C. L. Choy, "PZT ceramic/ceramic 0-3 nanocomposite films for ultrasonic transducer applications," Thin Solid Films 375, 95-99 (2000).

[4] R. Maas, M. Koch, N. R. Harris, N. M. White, and A. G. R. Evans, "Thick-film printing of PZT onto silicon," Materials Letters 31, 109-112 (1997).

[5] D. A. Payne and P. G. Clem, "Monolayer-mediated patterning of integrated electroceramics," J. Electroceram. 3, 163-172 (1999).

[6] R. A. Dorey, S. B. Stringfellow, and R. W. Whatmore, "Effect of sintering aid and repeated sol infiltrations on the dielectric and piezoelectric properties of a PZT composite thick film," J. Euro. Ceram. Soc. 22, 2921-2926 (2002).

[7] J. E. A. Southin, S. A. Wilson, D. Schmitt, and R. W. Whatmore, "e31, f determination for PZT films using a conventional 'd33' meter," J. Phys. D: Appl. Phys. 34, 1456-1460 (2001). 